\title{
Analysis of the upper massif of the craniofacial with the radial method - practical use
}

\author{
Tomasz Lepich', Józefa Dąbek², Daniel Stompel1', Jerzy S. Gielecki ${ }^{3}$
}

\begin{abstract}
1Department of Human Anatomy, Medical University of Silesia, Katowice, Poland 2Department of Cardiology, Medical University of Silesia, Katowice, Poland

3Department of Anatomy, Faculty of Medical Science, University of Varmia and Masuria, Olsztyn, Poland
\end{abstract}

Submitted: 1 February 2010

Accepted: 26 July 2010

Arch Med Sci 2011; 7, 5: 870-876

DOI: 10.5114/aoms.2011.25564

Copyright @ 2011 Termedia \& Banach

\begin{abstract}
Introduction: The analysis of the upper massif of the craniofacial (UMC) is widely used in many fields of science. The aim of the study was to create a high resolution computer system based on a digital information record and on vector graphics, that could enable dimension measuring and evaluation of craniofacial shape using the radial method.

Material and methods: The study was carried out on 184 skulls, in a good state of preservation, from the early middle ages. The examined skulls were fixed into Molisson's craniostat in the author's own modification. They were directed in space towards the Frankfurt plane and photographed in frontal norm with a digital camera. The parameters describing the plane and dimensional structure of the UMC and orbits were obtained thanks to the computer analysis of the function recordings picturing the craniofacial structures and using software combining raster graphics with vector graphics.

Results: It was compared mean values of both orbits separately for male and female groups. In female skulls the comparison of the left and right side did not show statistically significant differences. In male group, higher values were observed for the right side. Only the circularity index presented higher values for the left side.

Conclusions: Computer graphics with the software used for analysing digital pictures of UMC and orbits increase the precision of measurements as well as the calculation possibilities. Recognition of the face in the post mortem examination is crucial for those working on identification in anthropology and criminology laboratories.
\end{abstract}

Key words: craniofacial, orbit, analysis.

\section{Introduction}

Digital analysis of the upper massif of craniofacial (UMC) may be of interest for many scientific fields such as anthropology, medicine and biology [1-3]. The UMC corresponds anatomically with the medial level of the facial part of the cranium (Figure 1). Its main structure is formed by the orbit - the most complicated part of the facial cranium regarding its three dimensional shape and its physiological role.

When analysing the orbit, it is usually presented as a flat structure, yet it is extremely rarely examined as a dimensional structure, taking into consideration not only its height and width, but also its depth [4]. The

\author{
Corresponding author: \\ Tomasz Lepich MD, PhD \\ Department \\ of Human Anatomy \\ Medical University of Silesia \\ 18 Medyków \\ 40-252 Katowice-Ligota, \\ Poland \\ Phone: +48 322526487 \\ Fax: \\ E-mail: lepich@sum.edu.pl
}


research presents a computer method of analysing the UMC, and especially its principal part, which is the orbit, whose contour forms a basis for the analysis.

\section{Material and methods}

In the presented method, in order to assess the orbit variability, Mollison's craniostat was used according to the author's modification. Unlike a classic craniostat, Mollison's craniostat is constructed on a base with three stands equipped in their upper part with a screw grip to hold the cranium [5]. Two of the stands hold up the lateral part of the cranium and one infraoccipital grip holds up the posterior part. The grips move horizontally at any distance. In addition, in order to position the cranium on the Frankfurt line (this plane is established by horizontal lines, drawn at a tangent from the lower margin of both orbits to the upper one of the meatus acusticus externus on the lateral part of the cranium), the craniostat is equipped with an indicator moving vertically and horizontally, thus making it possible to indicate the lower margin of the orbit (Figure 2).

All the examined skulls were pictured in norma frontalis. The orbit was examined on the basis of its digital pictures taken with Camedia C4040 (high resolution camera, matrix $2272 \times 170$ ) and the obtained results were analysed digitally using an application combining vector graphics and raster graphics that constitute a non-referential method.

The application submitted in our work - DICA 2.0 - is based on digital analysis of two dimensional high resolution photos. The software makes full use of digital measurement techniques that, thanks to

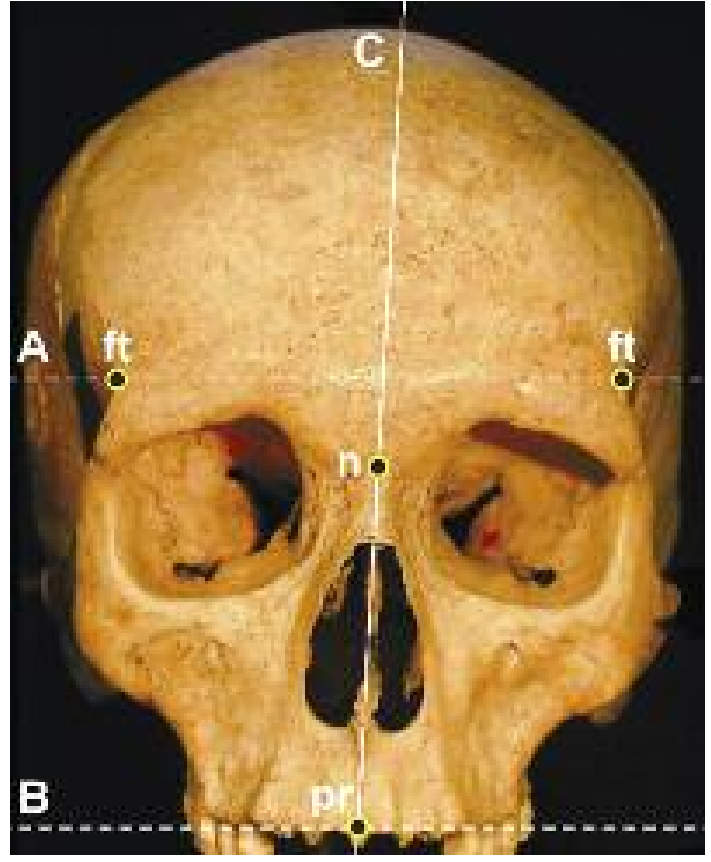

Figure 1. Evaluation of the upper part of the craniofacial limits

A-upper limit, $B$-lower limit, $C$ - median line of UMC, pr-prosthion, $n$-nasion, $f t$-frontotemporale

vector shape and orbit inlet size recording, help increase the precision of measurement to sub-pixel resolution. The DICA 2.0 application enables calculation of new qualitative parameters (functional for instance) as well as quantitative of the orbit (e.g. centre of gravity), that are impossible to define using classic anthropometric methods. It is also important to emphasize that the application detects asymmetry and deformation of the facial part of the cranium.

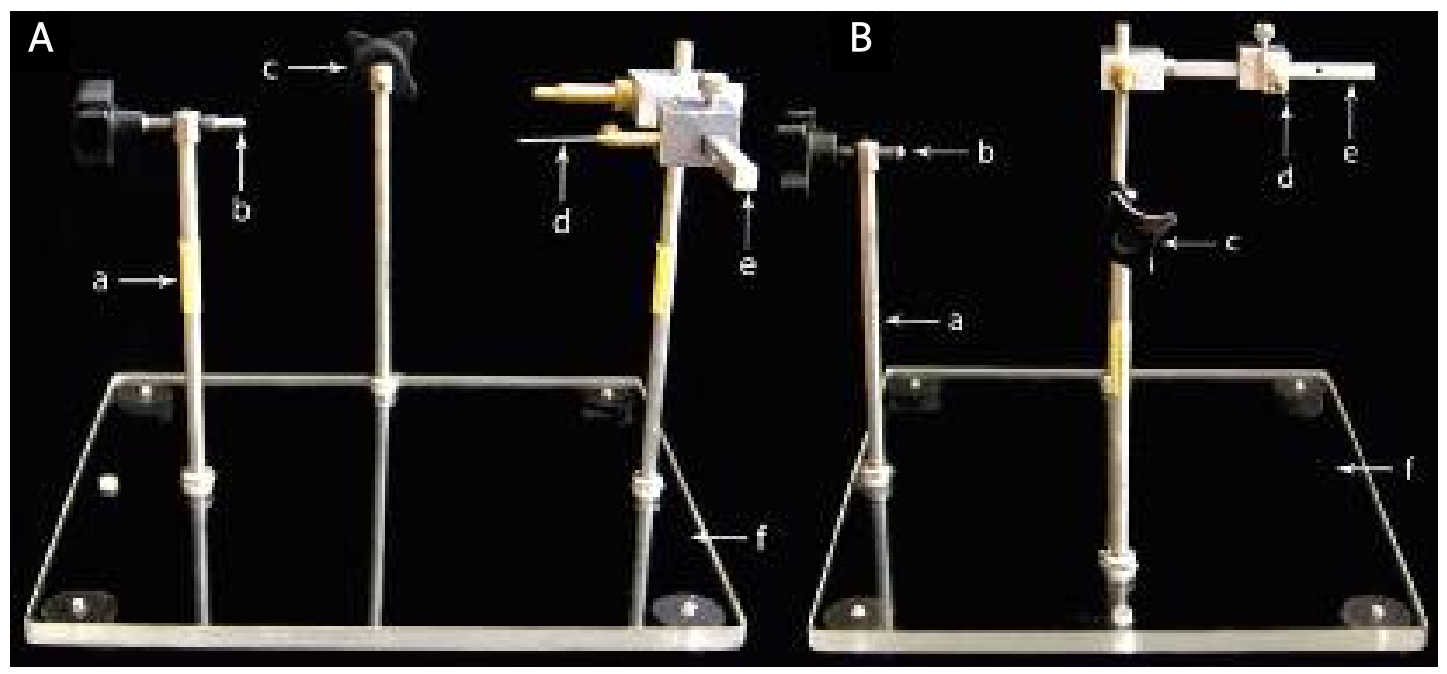

Figure 2. Craniostat

A-frontal view, $B$-lateral view, a-vertical beam, $b$-handle for the skull, $c$-suboccipital handle, $d$-the lower edge of the orbit's indicator, $e$-horizontal beam, $f$-base 


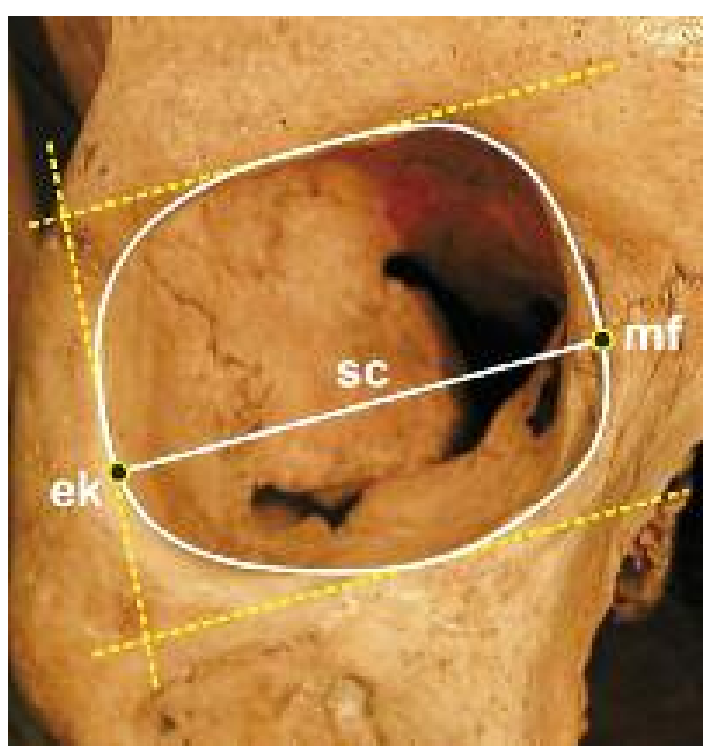

Figure 3. Marking of the $\mathrm{mf}$ and ek points on the orbit outline

mf-maxillofrontale, ek-ektokonchion, Sc-centre of gravity; accessory lines - interrupted, orbit outskirt - continuous

The skulls were examined using digital photos of them, marking unit segments on them. Then, the contour outlines of both orbits were drawn. The orbit contour was represented mathematically using spline functions that are formed from Bezier polynomials. The position of the mf point was marked next - an even point lying on the paracentral edge of the orbit. When outlining the orbit, an accessory line was marked, parallel to the upper or lower margin of the orbit, so that it may have as many tangent points to the margin as possible. It helps to define precisely the next anthropometric point - ek, which is found when drawing a parallel line to the accessory line, passing through the $\mathrm{mf}$ point. The ek point lies on the crossing of this line with the paracentral orbit edge (Figure 3).

The method of analysing pictures, based on a combination of raster and vector graphics, enables calibration and transposition of the pictures through various resolutions without changing the shape of the pictured objects. The pictures taken in raster graphics cannot be freely calibrated and for this reason it is important to take into consideration that applying only this method may lead to picture deformation. The method used in the presented work seems to be more objective and more precise than the classic anthropometric measurements.

The notion of the non-referential method (used interchangeably as a ray method) was taken from technical mechanics, where it is used to define a method of measuring shape deviation. The notion of shape deviation is applied in determination of the value that informs about the existing shape deviations between the examined profile and the reference profile. The average square circle (ASC), minimum circumscribed circle (MCC), maximum inscribed circle (MIC) or minimum zone circles (MZC - two concentric circles with a minimal radius difference) may all be defined as the reference profile. It is vital to remember, as it has been theoretically demonstrated, that the definitions of the MIC and MZC elements do not always permit one to obtain an unmistakable result of circularity deviation measurement [6, 7]. Mathematical dependencies support the application of ASC as the elements of reference as they are less susceptible to the influence of the position of the singular profile points and so they form an appropriate basis for determining multiple parameters of the examined profile.

The non-referential method was employed to examine the characteristics of the profiles formed by the orbit. It enables one to determine shape deviations for each radius, to find their minimal and maximal values, to calculate mean standard deviation and circularity deviation.

On the basis of the obtained orbit profile, it is possible to get information about its surface, circumference, width and height, maximal breadth calculated in relation to the maxillofrontal (mf) point and other characteristic values of its mathematical functional.

Practical use of the non-referential method does not take in consideration the real contour of the orbit, but its contour reduced photographically to its rectangular projection. Additionally, the contour should be mathematically specified by means of "glued" functions defined from Bezier polynomials of a general formula presented below:

$$
B(t)=\sum_{k=0}^{N} p_{k} \frac{N !}{k !(N-k) !} t^{k}(1-t)^{N-k}
$$

admitting that $N=4$, meaning third degree polynomials $(N-1$ defines the degree of a polynomial). The " $t$ " parameter adopts the values from the closed interval from 0 to 1 . The correct representation of the outline is equivalent to the outline in reality. There is a necessity for simultaneous use of raster graphics (being the basic one in computer systems) and vector graphics that give high precision of numerical calculations thanks to plotting Bezier curves on the photos. When defining the precision scale (which informs about the increase of the " $t$ " parameter in the interval $[0,1])$ there are from 1 to $n$ points resulting from equations for one raster point. However, the real number of points also depends on the screen resolution, to which it is inversely proportional. 
The algorithm for defining the centre of gravity coordinates for the "glued" curve that is based on the Bezier curve uses a parametric formula of the equation for a curve:

$\mathrm{B}(t)=\mathrm{B}(t)=\mathrm{PO}(1-t)^{3}+\mathrm{P}_{1} 3 t(1-t)^{2}+\mathrm{P}_{2} 3 t^{2}(1-t)+\mathrm{P}_{3} t^{3}$.

It is also based on the formulae given below:

$$
x=\frac{\sum_{i=1}^{n} m_{i} x_{i}}{\sum_{i=1}^{n} m_{i}},
$$$$
y=\frac{\sum_{i=1}^{n} m_{i} y_{i}}{\sum_{i=1}^{n} m_{i}} .
$$

The Bezier curve is treated as a system of material points $\mathrm{Mi}\left(x_{i}, y_{i}\right)$ which take the mass of a unit. The number of points admitted for calculation is equal to the number of points in the raster representation of the Bezier curve (the so-called curve shape is developed). It guarantees correctness of results independently of the shape and size of the curve and of the number of partial polynomials for the curves of continuous derivative of the first and higher degree. The centre of gravity of the examined contour is determined for the calculated points according to the following formulae:

$$
x=\frac{\sum_{i=1}^{n} x_{i}}{n}, \quad y=\frac{\sum_{i=1}^{n} y_{i}}{n} .
$$

Next, the coordinates of the centre of gravity are used to establish the distance between the points on the orbit contour and the centre of gravity. Here, a discrete model is employed. This model is developed for a series of 360 values

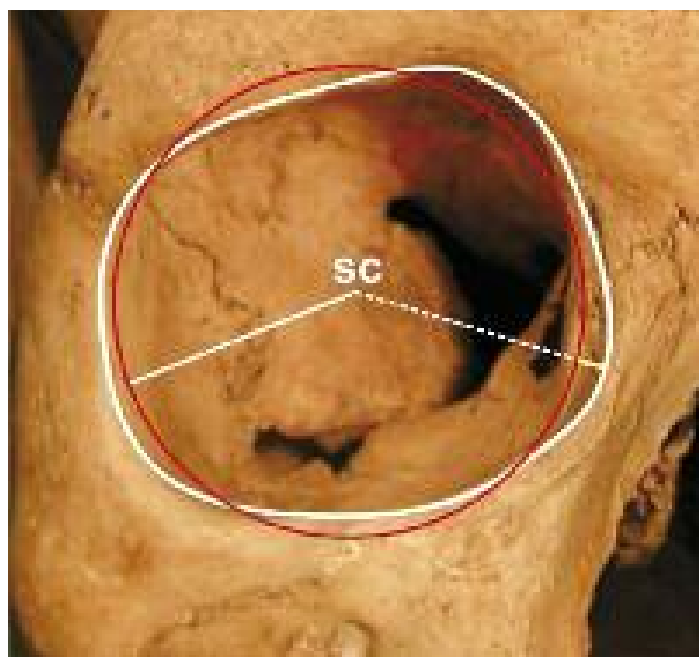

Figure 4. The highest elevation of the examined outline in relation to ASC

sc - centre of gravity; highest elevation (Amax) corresponding to angles from $0^{\circ}$ to $359^{\circ}$, which can be shown as:

$$
\varphi_{i}=\frac{2 \pi}{360} \times(i-1)
$$

where: $i=1,2 \ldots, 360$.

The analysed series of data allow for definition of the following values:

- the value for the reference circle (ASC - average square circle):

$$
r_{s r}=\frac{1}{n} \sum_{i=1}^{n} r_{i}
$$

- the value for the functional putting a numeric value to each contour:

$$
F=\sum_{i=1}^{n}\left(r_{i}-r_{s r}\right)^{2}
$$

- the highest elevation of the examined contour in relation to ASC (Figure 4):

$$
A_{\max }=\max \left\{\Delta r_{i}\right\}
$$

- the deepest excavation towards the ASC (Figures $5,6)$ :

$$
A_{\min }=\left|\min \left\{\Delta r_{i}\right\}\right|, Z=A_{\max }+A_{\min },
$$

where: $\Delta r_{i}=r_{i}-r_{s r}$.

\section{Results}

We have compared mean values of both orbits separately for male and female groups. Our research on male skulls and the comparison of both orbits

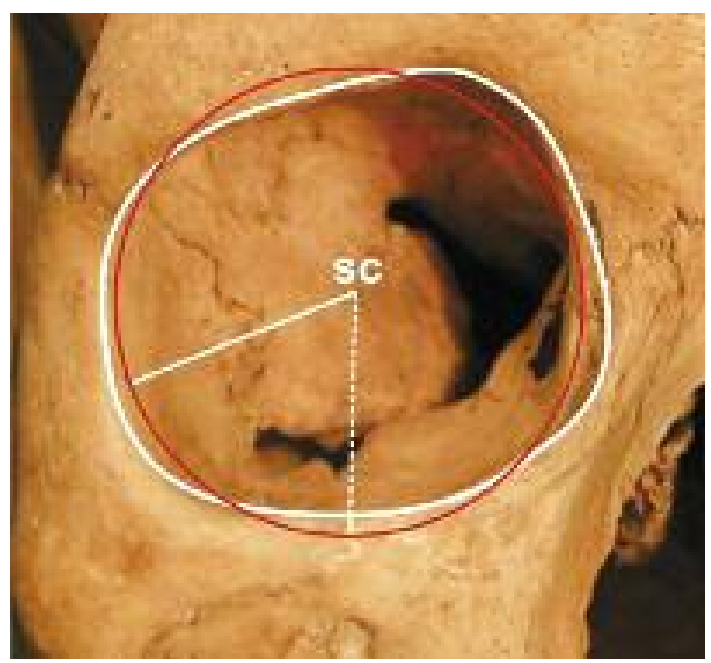

Figure 5. The deepest excavation towards the ASC sc - centre of gravity, deepest excavation (Amin) 


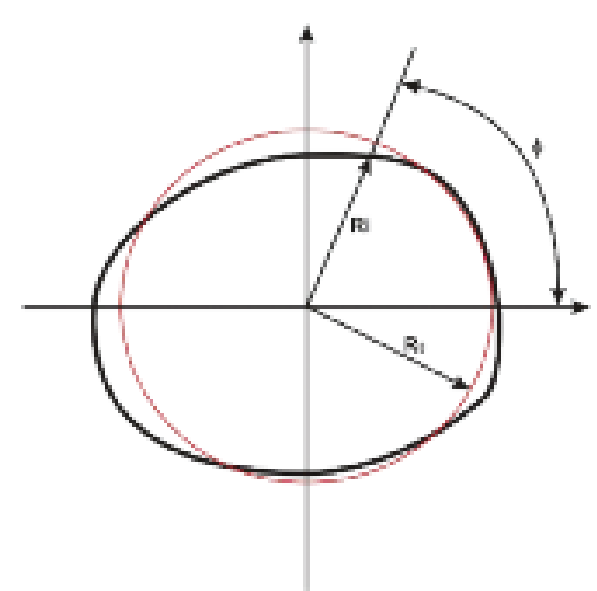

Figure 6. Discrete model of the orbit's outline $\phi$-angle in the range $\langle 0,359\rangle, R i-$ momentary radius of the examined outline, $R o$-radius of the reference circle

permits us to state that there are statistically significant differences for: circle radius $(R)$, width (Smf-ek), highest elevation (Amax), deepest excavation (Amin), circularity deviation (the deviation of the orbit roundness) (Z), functional (F), circularity index (K), and surface area (P). Parameters such as orbit circumference, orbit height and the deepest excavation were not statistically significant. In the male group, higher values were observed for the right side. Only the circularity index $(\mathrm{K})$ presented higher values for the left side (Table I).

In female skulls the comparison of the left and right side did not show statistically significant differences. Only the following parameters were different: $\mathrm{O}, \mathrm{Hmf}$-ek. The circularity index (K) on the left side was of higher value. Other parameters were higher for the right side (Table II).

Table I. Left orbit (LO) and right orbit (RO) size and shape parameters in male skulls

\begin{tabular}{|lccc|}
\hline & RO & LO & Value of $p$ \\
\cline { 2 - 3 } & $X$ & $X$ & \\
\hline $\mathrm{P}\left[\mathrm{mm}^{2}\right]$ & 1178.31 & 1139.1 & 0.026 \\
\hline $\mathrm{O}[\mathrm{mm}]$ & 124.76 & 123.25 & 0.106 \\
\hline $\mathrm{R}[\mathrm{mm}]$ & 19.29 & 18.94 & 0.016 \\
\hline Smf-ek $[\mathrm{mm}]$ & 39.75 & 38.56 & 0.001 \\
\hline Hmf-ek $[\mathrm{mm}]$ & 34.12 & 33.87 & 0.412 \\
\hline Amax $[\mathrm{mm}]$ & 3.22 & 2.83 & 0.001 \\
\hline Amin $[\mathrm{mm}]$ & 2.87 & 2.66 & 0.030 \\
\hline Z $[\mathrm{mm}]$ & 5.93 & 5.40 & 0.001 \\
\hline K $[\mathrm{mm}]$ & 1.05 & 1.06 & 0.001 \\
\hline $\mathrm{F}$ & 2.86 & 2.41 & 0.003 \\
\hline
\end{tabular}

$X$ - arithmetic mean, $p$ - significance level. Statistically significant values of Student's t-test and Mann-Whitney $U$ test $(p<0.05)$
The comparison of male and female skulls enabled the following observations: there is a difference in the value of the left and right side functional only in the male group, whereas in female skulls differences were noted for left and right side maximal excavation in relation to the mean circle. The variables describing the orbit height did not show any differences for left and right side in all the examined skulls, irrespective of their sex. However, we have observed that all the parameters of the orbit size and shape were of higher values on the right side, except for the parameter K (Table III).

The comparison of the above-presented values calculated separately for the left and the right orbit leads to the conclusion about the orbit asymmetry.

The difference in roundness deviation and the difference between the value of the functionals make it possible to define which orbit has more differentiating shape features compared to the shape features of the reference circle. It should be additionally noted that the difference in values of the reference circle radius points to the larger orbit.

\section{Discussion}

Digital analysis of the UMC is a modern issue, but is poorly represented in the anthropological literature. Until now, measurement of the UMC parameters was carried out using classic anthropometric techniques. These measurements based on traditional methods are burdened with mistakes resulting from the way instruments are constructed (objective error) and also from the investigator's experience (subjective error) [8, 9]. Thus, the attempt to create a computer application based on digital image analysis that could assure high precision and repeatability when measuring the

Table II. Left orbit (LO) and right orbit (RO) size and shape parameters in female skulls

\begin{tabular}{|lccc|}
\hline & RO & LO & Value of $p$ \\
\cline { 2 - 3 } & $X$ & $X$ & \\
\hline$P\left[\mathrm{~mm}^{2}\right]$ & 1120.22 & 1086.77 & 0.021 \\
\hline O $[\mathrm{mm}]$ & 121.5 & 120.12 & 0.082 \\
\hline $\mathrm{R}[\mathrm{mm}]$ & 18.67 & 18.41 & 0.012 \\
\hline Smf-ek $[\mathrm{mm}]$ & 38.26 & 36.88 & 0.000 \\
\hline Hmf-ek $[\mathrm{mm}]$ & 33.78 & 33.85 & 0.787 \\
\hline Amax $[\mathrm{mm}]$ & 3.13 & 2.77 & 0.001 \\
\hline Amin $[\mathrm{mm}]$ & 2.42 & 2.21 & 0.009 \\
\hline Z $[\mathrm{mm}]$ & 5.54 & 4.99 & 0.001 \\
\hline K $[\mathrm{mm}]$ & 1.05 & 1.06 & 0.001 \\
\hline F & 2.42 & 1.92 & 0.012 \\
\hline
\end{tabular}

$x$-arithmetic mean, $p$ - significance level. Statistically significant values of Student's t-test and Mann-Whitney $U$ test $(p<0.05)$ 
UMC variability with special emphasis on the orbits' parameters of qualitative and quantitative value.

The analysis based on morphometric features resulting from digital image processing gives much more objective and reliable data in comparison to the measurements carried out with classic anthropometric methods. Another advantage of digital analysis is its rapidity and ease of performing calculations. The accessible literature confirms that digital analysis is of better efficiency than classic measurement methods.

The craniometric research by Hildebolt et al. [10] performed on classic and digital data (using the Unigraphics CAD/CAM system) confirms the efficiency of digital methods for craniometric measurement. In addition, the rapidity and ease of digital measurement have been underlined by the cited authors.

Lascala et al. [11] carried out a 13-parameter analysis using classic craniometric methods such as slide caliper. Then, the skulls were scanned with conical beam computed tomography (CBCT). The survey confirms the efficiency of digital analysis in measuring the external surface of the skull.

Similarly, Cavalcanti et al. [12] also point out that the digital method (3D-CT) is more precise and accurate than the classic one. Park et al. [13] compared in turn classic anthropometric photographs of skulls with the measurement based on 3D laser. The examples given above support the thesis that digital analysis is accurate and useful for clinical and scientific research.

The computer application "DICA 2.0" that we would like to present is based on digital analysis of 2D high resolution photographs. The software makes full use of digital measurement techniques that enable the researchers to increase precision to sub-pixel resolution thanks to vector recording of the shape and size of the orbit inlet parameters. Regarding the anatomical features of the orbit margin, especially its zygomatic part, it is admitted that the measurement error exceeds $0.5 \mathrm{~mm}$.

For other parts of the cranium the error is $1 \mathrm{~mm}$. Outlining the orbit is burdened with difficulties such as orbit margin irregularities and concurrent structures (supraorbital incision for instance). The orbit outline irregularity is eliminated with the use of Bezier curves. This method informs about the difference in placement of clinically important areas on the orbit margin (supraorbital or frontal incision). Outlining the orbit starting from the mf point makes possible the orbit comparison, which is important for clinical and criminalistic research.

It is also worth emphasizing that the application makes it possible to detect small deformations and asymmetry of the facial cranium.

DICA 2.0 may be used as a database and catalogue of the examined material and para-
Table III. Left orbit (LO) and right orbit (RO) size and shape parameters without separating sexes

\begin{tabular}{|lccc|}
\hline Parameter & RO & LO & Value of $p$ \\
\cline { 2 - 3 } & $X$ & $X$ & \\
\hline $\mathrm{P}\left[\mathrm{mm}^{2}\right]$ & 1153.37 & 1116.63 & 0.006 \\
\hline O $[\mathrm{mm}]$ & 123.36 & 121.91 & 0.047 \\
\hline $\mathrm{R}[\mathrm{mm}]$ & 19.02 & 18.71 & 0.005 \\
\hline Smf-ek $[\mathrm{mm}]$ & 39.11 & 37.84 & 0.001 \\
\hline Hmf-ek $[\mathrm{mm}]$ & 33.98 & 33.86 & 0.619 \\
\hline Amax $[\mathrm{mm}]$ & 3.09 & 2.75 & 0.001 \\
\hline Amin $[\mathrm{mm}]$ & 3.18 & 2.81 & 0.001 \\
\hline Z $[\mathrm{mm}]$ & 5.76 & 5.22 & 0.001 \\
\hline K $[\mathrm{mm}]$ & 1.05 & 1.06 & 0.001 \\
\hline F & 2.67 & 2.20 & 0.001 \\
\hline
\end{tabular}

$X$ - arithmetic mean, $p$ - significance level, $R O$ - right orbit, $L O$ - left orbit. Statistically significant values of Student's t-test and MannWhitney $U$ test $(p<0.05)$

meters. Digital recording of photographs enables multiple measurements and creates an accessible database for other researchers. The presented DICA 2.0 version however is not adapted to process files recorded in the DICOM format that is widespread in image diagnostics.

There is a possibility to convert the files in DICOM format, so that the DICA 2.0 application receives data in the appropriate form for further analysis. Thanks to this possibility, calculating the orbit inlet parameters as well as the craniofacial parameters may be of use for radiologists and surgeons.

The method of combining raster and vector graphics makes it possible to calibrate and transfer pictures of different resolution without changing the shape of the analysed object. This is a method of higher precision and objectiveness than the classic anthropometric method.

For the purpose of digital orbit analysis we have applied the non-referring method (radial method) based on shape deviation analysis of the orbit shape in relation to the model circle as a reference profile.

The method was applied to examine characteristics of the profiles formed by all the orbits. It examines the values of local shape deviations for a given radius; it finds their minimal and maximal values as well as the values of the circularity deviations, the highest elevation and the deepest excavation. In addition, it makes it possible to compare the left and right orbit, to perform a harmonic characteristic of the profiles formed by the orbit outlines.

On the basis of the orbit profile we can obtain information about its surface, its circumference length, maximal and minimal width and about the 
characteristic values of the functionals. The nonreferring method reduces the real orbit outline to the outline represented when photographing the orbit from the rectangular projection.

This outline is then mathematically specified using the so-called glued function defined from Bezier polynomials. In order to represent the orbit shape it is vital to apply raster graphics of high resolution, which is the basic representation for all orbit images in most computer applications, as well as vector graphics, which give high precision of numeral calculation thanks to the transposition of Bezier curves on the obtained photos.

The Bezier curves reject any deficient orbit outline measurement. The method points out the difference in placing clinically important points on the orbit circumference (such as supraorbital or frontal notch). The orbit circumference started from the $\mathrm{mf}$ point is helpful in comparing the orbit's characteristic parameters for the purpose of their clinical examination or for the use of criminology laboratories. When calculating the centre of gravity coordinates of the orbit, it is now possible to state what is the distance separating the centre of gravity from the given points placed on the orbit circumference.

Median circle, maximal and minimal excavation and elevation, circularity deviation of each value all these constitute data that can be compared in order to conclude about the orbit asymmetry. Difference in circularity deviation as well as the functional value serve to establish which orbit is different in shape from the model circle. The skull has to be positioned in the same way for all measurements, so it is fixed into a craniostat. We have used Mollison's craniostat in our own modification, because of the fact that the photographs were taken from the perspective of norma frontalis.

In conclusion, digital image analysis based on the raster to vector image recording (semiautomatic) can supply fields of anthropological and medical research with many new parameters for the description and classification of the craniofacial area and the orbit, thus combining classical skull studies with the precision and objectivity of computer measurement techniques.

To sum up, the analysis of the upper massif of craniofacial is used in many scientific fields such as anthropology [14], criminology and maxillofacial surgery [15]. The examination of cranial asymmetry allows one to define multiple parameters characterizing the skeleton, which, in turn, helps restore the appearance of the face. The recognition of the face in postmortem examinations is crucial for the identification process and work of anthropology surgery and forensic laboratories [16-18].

\section{References}

1. Wanyura H. Urazy szkieletu czaszkowo-twarzowego. In: Kryst L. Chirurgia szczękowo-twarzowa [Polish]. PZWL, Warszawa 2004; 224-5.

2. Hommerich CP, Riegel A. Measuring of the piriform aperture in humans with $3 \mathrm{D}-\mathrm{SSD}-\mathrm{CT}$ - reconstructions. Ann Anat 2002; 184: 455-9.

3. Wandee A, Dolly M, Roengsak S, Wannapa P. Supraorbital notch/foramen, infraorbital foramen and mental foramen in Thais. Anthropometric measurements and surgical relevance. J Med Assoc Thai 2006; 89: 675-81

4. Piasecki K, Reymond J, Wysocki J. Kształt wejścia do oczodołu - metodyka badań [Polish]. Antropologia a Medycyna i Promocja Zdrowia 1996; 2: 1-80.

5. Reymond J, Piasecki K, Wanyura H. Kraniopantograf przyrząd do badania kształtu oczodołu [Polish]. Czas Stomatol 1990; 18: 91-3.

6. Neugebauer $M$. Uncertainty analysis for roundness measurements by the example of measurement on a glass hemisphere, IOP Publishing Ltd 2001.

7. Moore D. Design considerations in multiprobe roundness measurement, OP Publishing Ltd 1989.

8. Piasecki K. Las nuevas escalas craneoscopicas. Observaciones preliminares. 1992 UW.

9. Perini TA, de Oliveira GL, dos Santos Ornellas J, de Oliveira FP. Technical error of measurement in anthropometry. Rev Bras Med Esporte 2005; 11: 86-90.

10. Hildebolt CF, Vannier MW. Three-dimensional measurement accuracy of skull surface landmarks. Am J Phys Anthropol 1988; 76: 497-503.

11. Lascala CA, Panella J, Marques MM. Analysis of the accuracy of linear measurements obtained by cone beam computed tomography (CBCT - NewTom). Dentomaxillofac Radiol 2004; 33: 291-4.

12. Cavalcanti MG, Rocha SS, Vannier MW. Craniofacial measurements based on 3D-CT volume rendering: implications for clinical applications. Dentomaxillofac Radiol 2004; 33: 170-6.

13. Park HK, Chung JW, Kho HS. Use of hand-held laser scanning in the assessment of craniometry. Forensic Sci Int 2006; 160: 200-6.

14. Lindenbaum S. Cannibalism, kuru and anthropology. Folia Neuropathol 2009; 47: 138-44

15. Rutkowska M, Gerber H, Nowak R, Piątkowski J. Depression in patients with oral or facial malignancy. Arch Med Sci 2007; 3: 392-5.

16. Gliddon MJ, Xia JJ, Gateno J, et al. The accuracy of cephalometric tracing superimposition. J Oral Maxillofac Surg 2006; 64: 194-202.

17. Dong Y, Zhao Y, Bai S, Wu G, Wang B. Three-dimensional anthropometric analysis of the Chinese nose. J Plast Reconstr Aesthet Surg 2010; 63: 1832-9.

18. Stephan CN, Davidson PL. The placement of the human eyeball and canthi in craniofacial identification. J Forensic Sci 2008; 53: 612-9. 